\title{
Normal-Dominated Singularities in Static Space-Times
}

\author{
E.P.T. Liang \\ Center for Relativity Theory, The University of Texas at Austin, Austin, Texas, USA
}

Received January 28, 1973

\begin{abstract}
We define "normal-dominated" singularities of static solutions of the Einstein equations and show that a uniquely and invariantly defined structure can be assigned to these singularities. We find for the general solution that the dominant term of the Riemann tensor near the singularity is of Petrov Type N. Except for one special class of solutions, it seems that in general the shear of the null geodesics blows up at the same rate as their convergence near the singularity, in contradistinction to the "elementary singularity" of Newman and Posadas. We compute the structure for a variety of known static solutions as well as the stationary Kerr-Newman metrics.
\end{abstract}

\section{Introduction}

The singularity theorems of Hawking, Penrose and others [1-4] indicate that, assuming reasonable properties for the matter source and global structure, singularity in general relativistic space-times is both a general and stable phenomenon. It is then natural to inquire about the nature and structure of these singularities. For the sake of physical interest, we will consider only the type for which curvature or matter variables blow up. In cosmological models ${ }^{1}$, the works of Lifshitz, Khalatnikov and Belinskii [5], and of Eardley, Liang and Sachs [6] seem to indicate that the singularities are of two types, namely, the velocity-dominated and the mix-master. Moreover, in the velocitydominated case, a unique and invariant structure can be assigned to the singularity. What about other space-times?

In this paper we are going to show that a similar construction can be worked out for the class of static space-times which we call "normaldominated." In a static ${ }^{2}$ space-time, the singularity, if it exists, must be in some sense "time-like," since it must be tangent to the time-like

* Supported in part by NSF Grant GP 34639 X.

1 By a cosmological model we mean a space-time which admits no time-like Killing vector and contains some form of macroscopic matter source.

${ }^{2}$ A space-time is static if it admits an irrotational Killing vector every-where time-like except maybe at the singularities. Thus Schwarzschild solution is not static near the singularity $r=0$. 
Killing vector field. Intuitively, we call a solution and its singularity "normal-dominated" if there exists a family of time-like hypersurfaces "parallel" to the singularity in some geodesic sense and whose intrinsic curvatures become small compared to their extrinsic curvatures in the field equations near the singularity. In this case if we drop the intrinsic curvature terms in the Einstein equations the remaining equations can be explicitly integrated to give the first order solution near the singularity. Some integration functions can then be identified as the singularity metric and extrinsic curvature. Most known static solutions studied turn out to be normal-dominated.

In Section II we define normal-dominated singularities of static space-times and exhibit their structures. In Section III we discuss the behaviors of geodesics and curvature components near the singularity. Two interesting results are obtained. First, we find that for null geodesics congruences near the singularity, except in very special cases, the shear seems to blow up at the same rate as the convergence. This is different from the "elementary singularity" of Newman and Posadas [7], in which case the null geodesics are shear-free near the singularity. Second, for most normal-dominated static solutions, the asymptotic Riemann tensor near the singularity has a dominant component which is Type N. This suggests that the Riemann tensor might also have special algebraic properties near a generic space-time singularity, just as they do in asymptotically flat regions. Some well known static solutions are studied in Section IV, and the singularity of the Kerr-Newman stationary solutions is studied along similar lines in Section V. In the Appendix we record the tedious computations of the Riemann tensor components.

\section{The Structure of the Singularity}

In terms of Gaussian Normal coordinates based on a family of time-like hypersurfaces, the metric of a general static space-time can be written as

$$
d s^{2}=d r^{2}-V^{2}\left(r, x^{A}\right) d t^{2}+g_{A B}\left(r, x^{A}\right) d x^{A} d x^{B} \quad A=1,2
$$

where $V \equiv\langle\partial / \partial t, \partial / \partial t\rangle^{\frac{1}{2}}$ is the norm of the time-like Killing vector. The remaining coordinate freedoms are $x^{A} \rightarrow x^{\prime A}\left(x^{B}\right)$ plus the arbitrariness in choosing the initial hypersurface $r=$ const. The static Einstein equations are

$$
\begin{gathered}
G_{0}^{0}=-(1 / 2)^{(3)} R=0 \\
G_{a b}={ }^{(3)} R_{a b}+V_{\mid a b} / V=0 \quad x^{0}=t, x^{a}=\left(x^{A}, r\right)
\end{gathered}
$$

where geometric quantities formed with the metric of $t=$ const hypersurfaces ${ }^{(3)} d s^{2}=g_{a b} d x^{a} d x^{b}=d r^{2}+g_{A B} d x^{A} d x^{B}$ have a superscript "(3)" 
and a stroke "|" denotes co-derivative with respect to $g_{a b}$. If we write

$$
\begin{gathered}
K^{A}{ }_{B}=\frac{1}{2} g^{A C} \partial_{r} g_{C B} \\
\Leftrightarrow K \equiv K_{A}^{A}=\partial_{r}(\ln \alpha), \quad \alpha \equiv\left(\operatorname{det} g_{A B}\right)^{\frac{1}{2}}
\end{gathered}
$$

as the extrinsic curvature of the $r=$ const 2-surfaces (with respect to the $t=$ const hypersurfaces), Eqs. (2) can be split into:

$$
\begin{gathered}
-2 G_{0}^{0}={ }^{(3)} R={ }^{(2)} R+2 K_{, r}+K^{2}+K_{B}^{A} K^{B}{ }_{A}=0 \\
G_{r}^{r}=K_{, r}+K^{A}{ }_{B} K^{B}{ }_{A}+V_{, r r} / V=0 \\
G_{A}^{r}=K_{, A}-K_{A \| B}^{B}+V_{, r A} / V-V_{, C} K_{A}^{C} / V=0, \\
G_{B}^{A}{ }_{B}^{(2)} R_{B}^{A}+K_{B, r}^{A}+K K_{B}^{A}+V^{, A}{ }_{\| B} / V+V_{, r} K_{B}^{A} / V=0,
\end{gathered}
$$

where geometric quantities formed with the 2-metric $g_{A B}$ have a superscript "(2)", a double stroke "\|" denotes co-derivative with respect to $g_{A B}$, and a comma "," denotes ordinary derivative.

Suppose we assume that in some region of space-time the terms involving $\partial / \partial r$ alone are large compared to the other terms in Eqs. (4) and (7), then we need only keep the $\partial / \partial r$ terms as a first order approximation:

$$
\begin{gathered}
2 K_{, r}+K^{2}+K^{A}{ }_{B} K_{A}^{B}=0, \\
K^{A}{ }_{B, r}+K K_{B}^{A}{ }_{B}+V_{, r} K_{B}^{A} / V=0 .
\end{gathered}
$$

Eq. (9) has the first integral

$K^{A}{ }_{B}={ }_{0} M^{A}{ }_{B}\left(x^{A}\right)(\alpha V)^{-1},{ }_{0} M^{A}{ }_{B}$ arbitrary functions of integration . (10)

Putting solutions (10) back into Eq. (8) we get

$$
\begin{gathered}
\alpha V={ }_{0} C\left(r-{ }_{0} r\right) \\
{ }_{0} C \equiv \frac{1}{2}\left({ }_{0} M^{2}+{ }_{0} M^{A}{ }_{B}{ }_{0} M^{B}{ }_{A}\right) /{ }_{0} M,{ }_{0} M \equiv{ }_{0} M_{A}^{A} \text { and }{ }_{0} r
\end{gathered}
$$

is another arbitrary function of integration.

Substituting (11) in (10) we can further integrate to obtain

$$
g_{A B}={ }_{0} g_{A C}\left(x^{D}\right) \exp \left\{2{ }_{0} K^{C}{ }_{B} \ln \left(r-{ }_{0} r\right)\right\}
$$

where ${ }_{0} g_{A C}$ 's are again arbitrary functions of integration and ${ }_{0} K^{C}{ }_{B}$ $\equiv{ }_{0} M_{B}^{C}{ }_{B} C$. If we define a parameter $\gamma \equiv{ }_{0} M^{A}{ }_{B 0} M^{B}{ }_{A} /{ }_{0} M^{2},\left(\infty \geqq \gamma \geqq \frac{1}{2}\right)$, then ${ }_{0} K^{C}{ }_{B}$ satisfies the constraints

$$
{ }_{0} K^{C}{ }_{C}=2 /(1+\gamma), \quad{ }_{0} K^{A}{ }_{B 0} K^{B}{ }_{A}=4 \gamma /(1+\gamma)^{2} .
$$


Using Eqs. (11) and (12) we now have a complete first order solution,

$$
\begin{aligned}
& \alpha={ }_{0} \alpha\left(r-{ }_{0} r\right)^{2 /(1+\gamma)} \quad{ }_{0} \alpha \equiv\left(\operatorname{det}_{0} g_{A B}\right)^{\frac{1}{2}}, \\
& V={ }_{0} V\left(r-{ }_{0} r\right)^{-(1-\gamma) /(1+\gamma)} \quad{ }_{0} V \equiv{ }_{0} C /{ }_{0} \alpha .
\end{aligned}
$$

Since ${ }_{0} g_{A C}$ is positive definite, and both ${ }_{0} g_{A B}$ and ${ }_{0} K_{A B} \equiv{ }_{0} g_{A C}{ }_{0} K_{B}{ }_{B}$ are symmetric tensors, ${ }_{0} K^{A}{ }_{B}$ and ${ }_{0} g_{A C}$ can be simultaneously diagonalized at each point by a real coordinate transformation. Furthermore, since in 2-dimensions any vector field is hypersurface orthogonal we can in fact choose orthogonal local coordinates tangent to the eigenvectors of ${ }_{0} K^{A}{ }_{B}$. If we write

$$
p_{ \pm}=\left(1 \pm(2 \gamma-1)^{\frac{1}{2}}\right) /(1+\gamma)
$$

as the eigenvalues of ${ }_{0} K^{A}{ }_{B}$ and let $x^{ \pm}$be the associated coordinates, then the first order solution can be put in the form

$$
\begin{aligned}
d s^{2}= & -{ }_{0} V^{2}\left(r-{ }_{0} r\right)^{2 p_{0}} d t^{2} \\
& +\sum_{A=+}{ }_{0} g_{A}\left(x^{B}\right)\left(r-{ }_{0} r\right)^{2 p_{A}}\left(d x^{A}\right)^{2} \quad p_{0} \equiv-(1-\gamma) /(1+\gamma) .
\end{aligned}
$$

The exponents $p_{0}, p_{ \pm}$as functions of $\gamma$ are plotted in Fig. 1. Computing the quadratic curvature invariant

$$
\begin{aligned}
& I \equiv(1 / 8) R_{\mu v \sigma Q} R^{\mu v \sigma Q}=V_{\mid a b} V^{\mid a b}=\left\{V_{\| A B} V^{\| A B}+2 V_{, r} K^{A}{ }_{B} V^{, B}{ }_{\| A}+V_{, r r}{ }^{2}\right. \\
& \left.+V_{, r}{ }^{2} K^{A}{ }_{B} K^{B}{ }_{A}+2 V_{, r A} V^{, A}{ }_{, r}-4 V_{, r A} V_{, B} K^{B A}+2 V_{, A} V_{, B} K^{A}{ }_{C} K^{C B}\right\} V^{-2}
\end{aligned}
$$

for solutions $\left(16^{\prime}\right)$ we find that $r={ }_{0} r$ indeed corresponds to a physical singularity $\left(I \sim\left(r-{ }_{0} r\right)^{-4}\right)$ except when $\gamma=\infty$, in which case $I$ is finite. Since $V$ vanishes in this case $r={ }_{0} r$ is just a regular Killing horizon (e.g., $r=2 m$ in Schwarzschild solution). We will not consider such cases and assume from now on $\infty>\gamma \geqq \frac{1}{2}$ so that $r={ }_{0} r$ is always singular. From Eq. (14) we see that there are three types of singularities:

(a) $\gamma>1, p_{0}>0, V \rightarrow 0$ as $r \rightarrow_{0} r$, the singularity corresponds to infinite red-shift for signals coming out of the singularity.

(b) $\gamma<1, p_{0}<0, V \rightarrow \infty$ as $r \rightarrow_{0} r$, the singularity corresponds to infinite blue-shift for signals coming out of the singularity.

(c) $\gamma=1, p_{0}=0, V$ finite as $r \rightarrow{ }_{0} r$.

In order that metric $\left(16^{\prime}\right)$ be a true first-order solution near the singularity, the consistency conditions

$$
\begin{aligned}
& { }^{(2)} R^{A}{ }_{B}\left(K^{C}{ }_{D} K^{D}{ }_{C}\right)^{-1} \rightarrow 0 \quad \text { as } \quad r \rightarrow{ }_{0} r \\
& V^{, A}{ }_{\| B} V^{-1}\left(K^{C}{ }_{D} K^{D}{ }_{C}\right)^{-1} \rightarrow 0 \quad \text { as } \quad r \rightarrow{ }_{0} r
\end{aligned}
$$




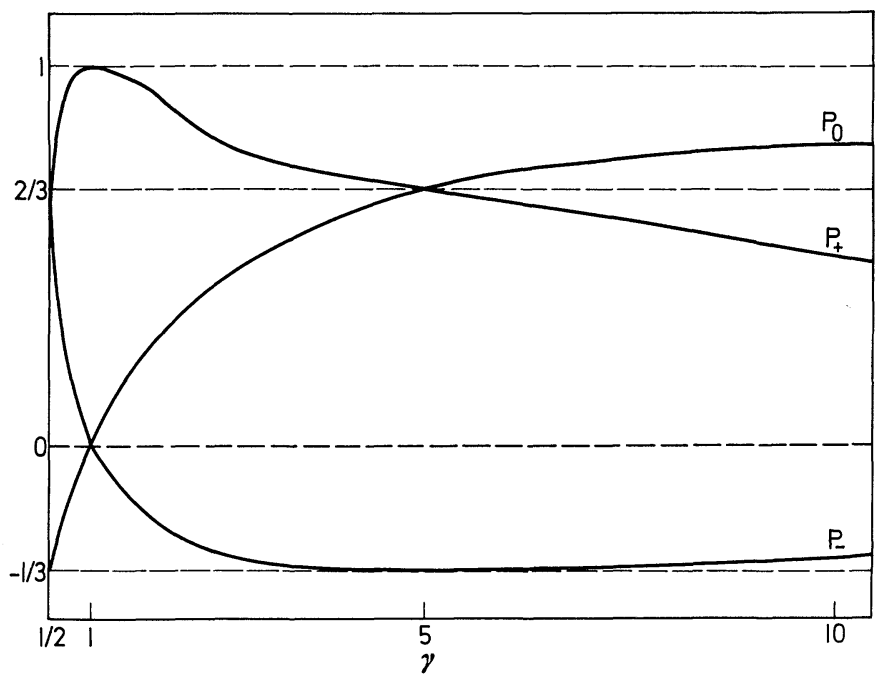

Fig. 1. Sketch of the three eigenvalues $\boldsymbol{P} \equiv\left(p_{0}, p_{+}, p_{-}\right)$as functions of the parameter $\gamma$

must be satisfied. Evaluating ${ }^{(2)} R^{A}{ }_{B}, V^{, A}{ }_{\| B} \cdot V^{-1}$ etc. using metric $\left(16^{\prime}\right)$, we find that Eqs. (18) are satisfied only when the conditions ${ }^{3}$

$$
{ }_{0}{ }^{r}, A=0 \text { whenever } \quad p_{A} \geqq 0, \quad A=+ \text { or }-.
$$

are imposed. Thus in principle we can still have ${ }_{0} r={ }_{0} r\left(x^{-}\right)$when $p_{-}<0$ (see Fig. 1). However, in this case one can show that there exists an asymptotic coordinate transformation $\bar{r}=r-{ }_{0} r\left(x^{-}\right)$such that the corresponding first-order solutions in a Gaussian coordinate system based on $\bar{r}=$ const hypersurfaces still satisfy the conditions $(18)^{4}$. Hence without lost of generality, we can always define $r=0$ as the singularity, and the coordinate system is rigidly fixed. Metric $\left(16^{\prime}\right)$ becomes

$$
d s^{2}=-{ }_{0} V^{2} r^{2 p_{0}} d t^{2}+\sum_{A=+}^{-}{ }_{0} g_{A} r^{2 p_{A}}\left(d x^{A}\right)^{2} .
$$

The geodesic congruence $\partial / \partial r$ is now the invariant geodesic congruence that hits the singularity orthogonally, which we call the singularity normal congruence, and the $r=$ const hypersurfaces are "parallel" to the singularity in this sense. Below we give an invariant characterization of the above procedure.

${ }^{3}$ When $\gamma=1$, the ${ }^{(2)} R$ terms are still of the same order as the $K$ terms even when the condition (19) is imposed, so that consistency requirements (18) are formally violated. Thus it might appear that there are no normal-dominated solutions with $\gamma=1$. However, for some mysterious reasons exact solutions with $\gamma=1$ do exist. So we will formally include them in this paper.

${ }^{4}$ Private communication from Dr. D. Eardley. 
Suppose that a static space-time has a singularity which locally admits a singularity normal congruence, then a Gaussian Normal coordinate system can be established invariantly with respect to this congruence and the metric can be written in the form (1), with $r=0$ chosen to be the singularity for convenience. Let $\left\{\hat{V}, \hat{g}_{A B}\right\}$ with corresponding $(\ln \hat{V})_{, r} \hat{K}_{A}{ }^{B}$ represent the exact solution in this coordinate system. Suppose there is another $\left\{V, g_{A B}\right\}$ which in some neighborhood of $r=0$ satisfies

(a) $g_{A B}=g_{B A}$, sgn. $g_{A B}=+2$;

(b) $\left\{V, g_{A B}\right\}$ obeys Eqs. (8) and (9);

(c) there exist some component, say $W$, of $\left\{V, g_{A B}\right\}$ such that for fixed $t, x^{A}$ and $r \rightarrow 0$

$$
(\hat{V}-V) W^{-1} \rightarrow 0, \quad\left(\hat{g}_{A B}-g_{A B}\right) W^{-1} \rightarrow 0
$$

$\left((\ln \hat{V})_{, r}-(\ln V)_{, r}\right)\left(K_{D}^{C}{ }_{D} K_{C}^{D}\right)^{-1} \rightarrow 0, \quad\left(\hat{K}_{B}^{A}{ }_{B}-K^{A}{ }_{B}\right)\left(K^{C}{ }_{D} K^{D}{ }_{C}\right)^{-1} \rightarrow 0$.

Then we call $\left\{\hat{V}, \hat{g}_{A B}\right\}$ and its singularity "normal-dominated" and $\left\{V, g_{A B}\right\}$ its first-order approximation. In order that a solution of Eqs. (8) and (9) be a first approximation, the conditions (18) (with ${ }_{0} r=0$ ) must be satisfied. Intuitively, this means that the intrinsic curvatures of the $r=$ const hypersurfaces are small compared to the extrinsic curvatures in the Einstein equations near the singularity.

Since the first-order 3-metric of the $r=$ const hypersurfaces can be put in the form (16) for all normal-dominated singularities, we now define

$$
{ }_{0} d s^{2}=-{ }_{0} V^{2} d t^{2}+\sum_{A=+}^{-}{ }_{0} g_{A}\left(d x^{A}\right)^{2}
$$

to be the metric of the singularity manifold and $p \equiv\left(p_{0}, p_{ \pm}\right)$to be its extrinsic curvature.

There remains the field Eqs. (5) and (6). To see what kind of additional constraints they impose on the first order solution let us consider the Bianchi Identities

$$
G_{\mu ; \nu}{ }^{v}=0, \quad \mu, v=0 \ldots 3 .
$$

Suppose that Eq. (7) is identically satisfied then (22) implies $G_{A}^{r}={ }_{0} G^{r}{ }_{A}\left(x^{A}\right)(\alpha V)^{-1}, \quad{ }_{0} G_{A}^{r} \quad$ arbitrary function of integration . (23) Furthermore, if (6) is identically satisfied then (22) implies $G_{r}^{r}={ }_{0} G_{r}^{r}\left(x^{A}\right)(\alpha V)^{-1}, \quad{ }_{0} G_{r}^{r} \quad$ arbitrary function of integration .

Thus in order that solutions (16) represent a first order solution to the full static Einstein equations it is necessary and sufficient that the $r^{-1}$ terms of $G_{A}^{r}$ and $G_{r}^{r}$ vanish identically. Putting solutions (16) into (5), the $r^{-2}$ 
term vanishes identically, but there remains a constraint on the higher order approximations which contribute to the $r^{-1}$ term. Putting solutions (16) into (6), the leading term, which is of order $r^{-1} \ln r$, vanishes identically, and the $r^{-1}$ term leads to the constraints:

$$
-p_{ \pm, \pm}+\left(\ln _{0} V\right)_{, \pm}\left(p_{0}-p_{ \pm}\right) \pm \frac{1}{2}\left(p_{-}-p_{+}\right)\left(\ln g_{\mp}\right)_{, \pm}=0
$$

where ${ }_{, \pm}$denotes $\partial / \partial x^{ \pm}$.

\section{Geodesics and the Riemann Tensor near the Singularity}

To study the behaviors of geodesics and curvature components of a normal-dominated static solution near the singularity, it suffices to consider the first order metric (16). Because of the arbitrary functions ${ }_{0} V\left(x^{ \pm}\right),{ }_{0} g\left(x^{ \pm}\right)$, the geodesic equations for the general case are not integrable. However, in the special case when both $\gamma$ and ${ }_{0} V$ are constants, $x^{ \pm}=$const are allowed solutions to the geodesic equation, and the corresponding geodesics (in the $r-t$ plane) are integrable. In the following we will restrict ourselves to this class of solutions.

(a) null geodesics:

With $x^{ \pm},{ }_{0} V, \gamma=$ constants, the geodesic equations reduce to

$$
\begin{aligned}
& \dot{t}={ }_{0} k / V^{2}, \quad{ }_{0} k=\text { const, “.” } \equiv d / d v \quad v \text { affine parameter. } \\
& \dot{r}={ }_{0} k / V
\end{aligned}
$$

The solution is

$$
\begin{gathered}
v={ }_{0} V_{0} k^{-1}((1+\gamma) / 2 \gamma) r^{2 \gamma /(1+\gamma)} \\
\left(t-{ }_{0} t\right)={ }_{0} V((1+\gamma) / 2) r^{2 /(1+\gamma)}
\end{gathered}
$$

with appropriate choice of the integration constants. A sketch of the null and time-like geodesics in the $r-t$ plane is given in Fig. 2.

(b) time-like geodesics:

In this case we still have $\dot{t}={ }_{0} k / V^{2}$, but now

$$
\dot{r}= \pm V^{-1}\left({ }_{0} k^{2}-V^{2}\right)^{\frac{1}{2}}
$$

and we have three distinct situations.

(1) $\gamma>1, V \rightarrow 0$ as $r \rightarrow 0$, all time-like geodesics can reach the singularity.

(2) $\gamma<1, V \rightarrow \infty$ as $r \rightarrow 0$, no time-like geodesics can reach the singularity. (A closer look at the full geodesic equations with the $x^{ \pm}$ terms shows that this is still true even when we allow $x^{ \pm}$to vary.) The manifold is time-like complete. In some sense, gravity is "repulsive" in this case.

(3) $\gamma=1, V=$ const, all geodesics with ${ }_{0} k^{2}>_{0} V^{2}$ can reach the singularity. 


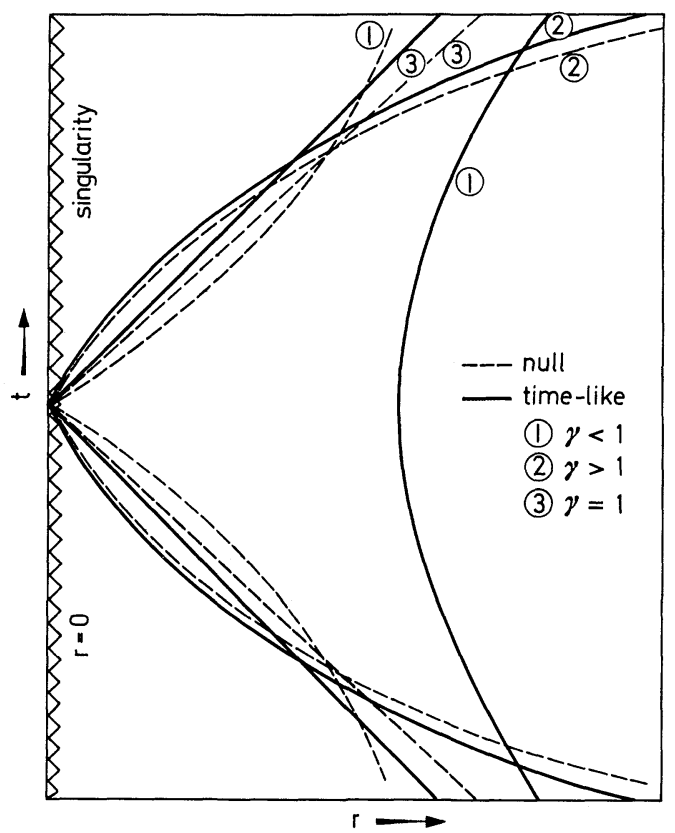

Fig. 2. Sketch of the time-like and null geodesics in the $r-t$ plane near the singularity $r=0$ for the three different cases $1 \gamma<1,2 \gamma>1,3 \gamma=1$

In cases (1) and (3), we have $\underset{r \rightarrow 0}{\simeq}$ const $\times V^{-1}$ and the behavior near the singularity is identical to that of the null geodesics (see Fig. 2).

Consider the null geodesic congruence tangent to the vector field $l^{\mu}=\dot{r} \delta_{r}^{\mu}+\dot{t} \delta_{t}^{\mu}$, which is normal to the null hypersurfaces

$$
u=t-\frac{1}{2}(1+\gamma) r^{2 /(1+\gamma)}=\text { const . }
$$

From Eq. (26) we have

$$
l^{\mu}={ }_{0} k V^{-1} \delta_{r}^{\mu}+{ }_{0} k V^{-2} \delta_{t}^{\mu} .
$$

The convergence of this geodesic congruence can be computed easily:

$$
\varrho \equiv-\frac{1}{2} l_{; \mu}^{\mu}=-{ }_{0} k_{0} V^{-1}(1+\gamma)^{-1} r^{-2 \gamma /(1+\gamma)}=-\frac{1}{2} \gamma^{-1} v^{-1} .
$$

On the other hand, if we put $p_{ \pm}=$const and ${ }_{0} V=$ const into the constraint Eqs. (25), we see that we must have

$$
\begin{aligned}
& \left(p_{+}-p_{-}\right)\left(\ln g_{\mp}\right)_{, \pm}=0 \\
& \Leftrightarrow \text { either (i) } p_{+}=p_{-} \Rightarrow \gamma=1 / 2, p_{ \pm}=2 / 3 \\
& \text { or (ii) } g_{\mp, \pm}=0 \text {. }
\end{aligned}
$$


In case (i) we have $\varrho=-v^{-1}, g_{A b}$ isotropic $^{5}$ :

$g_{A B} \sim{ }_{0} g_{A B} v^{2} \quad$ and $\quad \sigma \equiv\left(l_{(\mu ; v)} l^{(\mu ; v)}-\frac{1}{2}\left(l_{; \mu}^{\mu}\right)^{2}\right)^{\frac{1}{2}}=\frac{1}{\sqrt{2}}\left(p_{+}-p_{-}\right) v^{-1}=0$.

These resemble the singularities studied by Newman and Posadas ${ }^{6}[7]$. We see from above, however, that gravity is "repulsive" near the singularity for $\gamma<1$. Thus this case may not be very physical. In case (ii) we can redefine $x^{ \pm}$coordinates so that

$$
{ }^{(2)} d s^{2}=g_{A B} d x^{A} d x^{B}=r^{2 p+}\left(d x^{+}\right)^{2}+r^{2 p-}\left(d x^{-}\right)^{2}
$$

and the 2-spaces are flat, but in general anisotropic. If they are compact they must have toroidal topology (flat tori). Although we cannot explicitly integrate the geodesics in the generic case when both $\gamma$ and ${ }_{0} V$ are functions of $x^{ \pm}$, (so that $p_{+} \neq p_{-}$), from the above experience it seems that in general the shear of the null geodesics will blow up at the same rate as the convergence near a normal-dominated singularity, in contradistinction to the elementary singularity of Newman and Posadas [7], in which the null geodesics are asymptotically shear free. Since ours are "naked" singularities, whereas those of Newman and Posadas are in general believed to have event horizons, it appears that the asymptotic behavior of the shear of null geodesics near the singularity might have something to do with the existence of event horizons.

Let us now return to the general first-order metric (16) where $\gamma,{ }_{0} V$ are arbitrary functions of $x^{ \pm}$. The computation of the Riemann tensor components for this metric is rather tedious, and is recorded separately in the Appendix. Suppose we choose a null tetrad field near the singularity defined by

$$
\begin{aligned}
l^{\mu} & =1 / \sqrt{2}\left[V^{-1} \delta_{r}^{\mu}+V^{-2} \delta_{t}^{\mu}\right] ; \\
m^{\mu} & =1 / \sqrt{2}\left[g_{+}{ }^{-\frac{1}{2}} r^{-p_{+}} \delta_{+}^{\mu}+i_{0} g_{-}{ }^{-\frac{1}{2}} r^{-p-} \delta_{-}^{\mu}\right] \\
n^{\mu} & =1 / \sqrt{2}\left[-V \delta_{r}^{\mu}+\delta_{t}^{\mu}\right] ; \\
\bar{m}^{\mu} & =\text { complex conjugate of } m^{\mu}
\end{aligned}
$$

which satisfies the normalizations $-l^{\mu} n_{\mu}=+1=m^{\mu} \bar{m}_{\mu}, \quad l^{\mu} l_{\mu}=n^{\mu} n_{\mu}$ $=m^{\mu} m_{\mu}=0$, etc. Note that $l^{\mu}, n^{\mu}$ are in general not tangent to geodesics. It is straightforward to compute the five complex independent Weyl tensor components $\Psi_{0}, \Psi_{1}, \Psi_{2}, \Psi_{3}, \Psi_{4}[8]$, (which are just the Riemann tensor components since space-time is empty), with respect to this tetrad system. Using results from the Appendix and assuming $\gamma \neq 1$, we find

\footnotetext{
5 Throughout this paper " $\sim$ " means "the dominant term as $r \rightarrow 0$ is of the order of".

${ }^{6}$ It is not clear, however, whether the lowest non-vanishing term of $\sigma$ of our geodesics does behave as $\sim v$, as in the Newman-Posadas case.
} 


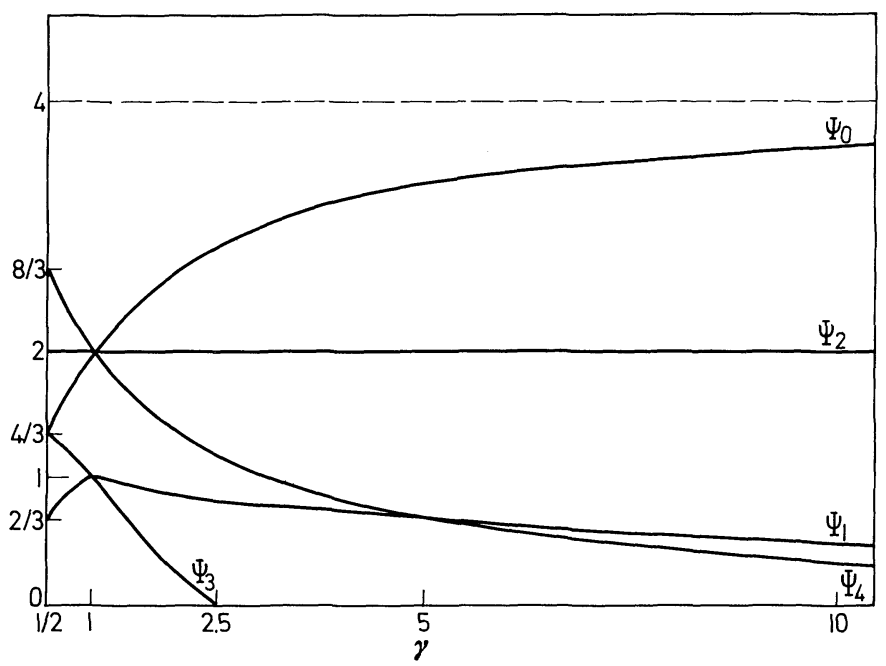

Fig. 3. Sketch of the largest (negative) exponents of the lowest order $\Psi$ 's as functions of $\gamma$. Note that for $\gamma \neq 1$ the exponent of either $\Psi_{0}$ or $\Psi_{4}$ is the largest. From this diagram one would like to conclude that the next dominant term is $\Psi_{2}$. However, it is not clear whether other $\Psi$ 's will be of the same order when the higher order contributions to metric (16) are taken into account

for the lowest order terms in the general case:

$$
\begin{aligned}
& \Psi_{0} \equiv-C_{\mu \nu \sigma \varrho} l^{\mu} m^{v} l^{\sigma} m^{\varrho} \sim r^{-4 \gamma /(1+\gamma)}+i r^{-2 \gamma /(1+\gamma)} \\
& \Psi_{1} \equiv-C_{\mu \nu \sigma \varrho} l^{\mu} n^{v} l^{\sigma} m^{\varrho} \sim r^{\left(-1-(2 \gamma-1)^{\frac{1}{2}}\right) /(1+\gamma)}+i r^{\left(-1+(2 \gamma-1)^{\left.\frac{1}{2}\right) /(1+\gamma)}\right.} \\
& \Psi_{2} \equiv-\frac{1}{2} C_{\mu \nu \sigma \varrho}\left(l^{\mu} n^{v} l^{\sigma} n^{\varrho}-l^{\mu} n^{v} m^{\sigma} \bar{m}^{\varrho}\right) \sim r^{-2} \text { independent of } \gamma \\
& \Psi_{3} \equiv-C_{\mu \nu \sigma \varrho} l^{\mu} n^{v} n^{\sigma} \bar{m}^{\varrho} \sim r^{\left(2 \gamma-3-(2 \gamma-1)^{\frac{1}{2}}\right) /(1+\gamma)}+i r^{\left(2 \gamma-3+(2 \gamma-1)^{\frac{1}{2}}\right) /(1+\gamma)} \\
& \Psi_{4} \equiv-C_{\mu \nu \sigma \varrho} n^{\mu} \bar{m}^{v} n^{\sigma} \bar{m}^{\varrho} \sim r^{-4 /(1+\gamma)}+i r^{(-4+2 \gamma) /(1+\gamma)}
\end{aligned}
$$

It must be pointed out, however, that some of coefficients of the above leading terms may vanish in special cases (i.e. for special functional forms or special values of ${ }_{0} V,{ }_{0} g_{ \pm}$and $\gamma$ ). In particular, for the special case discussed above, namely, when both ${ }_{0} V, \gamma$ are constants $(\Rightarrow \gamma=1 / 2)$, most of the coefficients of the leading terms are zero, and the first nonvanishing terms come from higher order contributions. But, putting aside these special cases, a closer look at the above leading exponents as functions of $\gamma$ (Fig. 3) reveals that for all values of $\gamma \neq 1$ or $1 / 2$, either $\Psi_{4}$ or $\Psi_{0}$ blows up fastest as $r \rightarrow 0$. Therefore the dominant term of the Riemann tensor near the singularity is either $\Psi_{4}$ or $\Psi_{0}$, depending on whether $\gamma<1$ or $\gamma>1$. We thus arrive at the interesting result: For $a$ 
general normal-dominated static space-time (with $\gamma \neq 1$ or $1 / 2$ ), the asymptotic Riemann tensor near the singularity has the dominant term belonging to type $N$, with principal null vector $l^{\mu}\left(n^{\mu}\right)$ when $\gamma<1(\gamma>1)$. We speculate, but have yet no concrete evidence, that the Riemann tensor near a generic singularity may also possess special algebraic properties.

\section{Examples}

In the following we look at some exact static solutions of the vacuum Einstein equations and analyze their singularity structure. The procedure, in principle, is to first construct a hypersurface orthogonal geodesic congruence that strikes the singularity orthogonally, $3+1$ split spacetime with respect to this congruence, and extract the singularity structure from the first order 3-metric of the "parallel" time-like hypersurfaces. In practice it is often not easy to construct the congruence explicitly, and we have to be satisfied with a structure obtained from using a nongeodesic congruence which seems to converge to the geodesic one near the singularity (i.e., their deviation from geodesics are of higher orders). The coordinates thus induced on the singularity manifold are therefore not the parameters of the orthogonal geodesics. But the structure itself should be equivalent. In many cases we are assured of the correctness of our procedure because the results obtained agree with the predicted forms (15) and (16).

(1) Cylindrical Solutions [9]:

$$
\begin{gathered}
d s^{2}=-e^{2 \alpha} d t^{2}+r^{2} e^{-2 \alpha} d \varphi^{2}+e^{2 \beta-2 \alpha}\left(d r^{2}+d z^{2}\right) \\
\alpha=c \ln r+d ; \quad \beta=c^{2} \ln r+k, \quad c, d, k \text { consts } .
\end{gathered}
$$

Singularity metric:

$$
{ }_{0} d s^{2}=-{ }_{0} V^{2} d t^{2}+{ }_{0} g_{z} d z^{2}+{ }_{0} g_{\varphi} d \varphi^{2}
$$

where ${ }_{0} V,{ }_{0} g_{z},{ }_{0} g_{\varphi}$ are messy functions of $c, d$, and $k$.

Extrinsic curvature:

$$
\boldsymbol{p}=\left(p_{0}, p_{+}, p_{-}\right) ; \gamma=\left(c^{2}+1\right) /(c-1)^{2} \quad c=0,1 \text { gives flat space-time . }
$$

(2) Plane Symmetric Solutions [10]:

$$
\begin{gathered}
d s^{2}=-\frac{m^{2}}{z} d t^{2}+z^{2}\left(d x^{2}+d y^{2}\right)+\frac{z d z^{2}}{m^{2}} \\
{ }_{0} d s^{2}=-m^{1 / 3}(3 / 2)^{-2 / 3} d t^{2}+(3 / 2)^{4 / 3} m^{4 / 3}\left(d x^{2}+d y^{2}\right) \\
\boldsymbol{p}=(-1 / 3,2 / 3,2 / 3) ; \quad \gamma=1 / 2 .
\end{gathered}
$$


(3) Weyl-Levi-Civita Axisymmetric Solutions [11]:

The general Weyl-Levi-Civita solutions have the metric

$$
d s^{2}=-e^{2 \psi} d t^{2}+e^{2 \chi-2 \psi}\left(d \varrho^{2}+d z^{2}\right)+e^{-2 \psi} \varrho^{2} d \varphi^{2} .
$$

where $\psi$ satisfies the cylindrical Laplace equation

$$
\frac{1}{\varrho} \frac{\partial}{\partial \varrho}\left(\varrho \frac{\partial}{\partial \varrho} \psi\right)+\frac{\partial^{2}}{\partial z^{2}} \psi=0
$$

and $\chi$ is related to $\psi$ through

$$
\begin{aligned}
& \chi_{, \dot{e}}=\varrho\left(\psi_{, \varrho}{ }^{2}-\psi_{, z}{ }^{2}\right) \\
& \chi_{, z}=2 \varrho \psi_{, \varrho} \psi_{, z} .
\end{aligned}
$$

We will concentrate on those solutions which are asymptotically flat. Because of axisymmetry, there are essentially three types of singularities:

(a) those which are singular on a continuous region of the $z$-axis;

(b) those which are singular on rings away from the $z$-axis;

(c) those which are singular on isolated points of the $z$-axis.

We now study examples of each class.

(a) To study solutions with a "rod-singularity" on the $z$-axis of the Weyl coordinates, it is more convenient to work in prolate spheroidal (P.S.) coordinates $(\lambda, \mu)$ :

$$
\begin{array}{ll}
\varrho=m\left(\lambda^{2}-1\right)^{\frac{1}{2}}\left(1-\mu^{2}\right)^{\frac{1}{2}} & m \text { any const } \neq 0 \\
z=m \lambda \mu & \infty \geqq \lambda \geqq 1,1 \geqq \mu \geqq-1 .
\end{array}
$$

The metric (33) then reads

$$
\begin{aligned}
d s^{2}=-e^{2 \psi} d t^{2} & +m^{2} e^{2 \chi-2 \psi}\left(d \lambda^{2} /\left(\lambda^{2}-1\right)+d \mu^{2} /\left(1-\mu^{2}\right)\right) \\
& +m^{2} e^{-2 \psi}\left(\lambda^{2}-1\right)\left(1-\mu^{2}\right) d \varphi^{2} .
\end{aligned}
$$

The Weyl equation in these coordinates

$$
\left(\left(\lambda^{2}-1\right) \psi_{, \lambda}\right)_{, \lambda}+\left(\left(1-\mu^{2}\right) \psi_{, \mu}\right)_{, \mu}=0
$$

has a general solution

$$
\psi=\sum_{l} q_{l} P_{l}(\mu) Q_{l}(\lambda)
$$

where $P_{l}, Q_{l}$ are the Legendre polynomials of the first and second kind. $\chi$ is then computable from

$$
\begin{aligned}
\chi_{, \lambda}= & \left(1-\mu^{2}\right)\left(\lambda^{2}-\mu^{2}\right)^{-1} \\
& \cdot\left\{\lambda\left(\lambda^{2}-1\right) \psi_{, \lambda}{ }^{2}-\lambda\left(1-\mu^{2}\right) \psi_{, \mu}{ }^{2}-2 \mu\left(\lambda^{2}-1\right) \psi_{, \lambda} \psi_{, \mu}\right\} \\
\chi_{, \mu}= & \left(\lambda^{2}-1\right)\left(\lambda^{2}-\mu^{2}\right)^{-1} \\
& \cdot\left\{\mu\left(\lambda^{2}-1\right) \psi_{, \lambda}{ }^{2}-\mu\left(1-\mu^{2}\right) \psi_{, \mu}{ }^{2}+2 \lambda\left(1-\mu^{2}\right) \psi_{, \lambda} \psi_{, \mu}\right\} .
\end{aligned}
$$


In particular, $\psi_{0}=P_{0} Q_{0}=\frac{1}{2} \ln \frac{\lambda-1}{\lambda+1}$ gives Schwarzschild solution. If we now consider a solution $\psi$ made up of a finite sum of the $P_{l} Q_{l}$ terms, then since $Q_{l}(\lambda)$ is singular at $\lambda=1$ for each $l, \psi$ is singular at $\lambda=1$, which is a "rod" extending from $z=-m$ to $z=+m$. One can check that except when $\psi=\psi_{0}, \lambda=1$ indeed corresponds to a physical singularity of the metric. We study these singularities in more detail.

Putting $\varepsilon=\lambda-1$, we find that

$$
\begin{aligned}
& \underset{\varepsilon \rightarrow 0}{\simeq} \frac{1}{2}(\ln \varepsilon) Q \quad Q(\mu)=\sum q_{l} P_{l}(\mu) \\
\Rightarrow & e_{\varepsilon \rightarrow 0} \underset{\varepsilon \rightarrow 0}{\simeq} \varepsilon^{Q / 2} .
\end{aligned}
$$

Using Eq. (37b) we find

$$
e_{\varepsilon \rightarrow 0}^{\gamma} \underset{\varepsilon}{\simeq} \varepsilon^{Q^{2} / 2}
$$

so that

$$
e^{\gamma-\psi} \underset{\varepsilon \rightarrow 0}{\simeq} \varepsilon^{Q(Q-1) / 2} .
$$

For $\mu \neq 1$ and $\varepsilon \rightarrow 0$ the metric (36) reduces to

$$
\begin{aligned}
d s^{2} \underset{\varepsilon \rightarrow 0}{\simeq}-\varepsilon^{Q} d t^{2} & +m^{2} \varepsilon^{Q(Q-1)}\left(1-\mu^{2}\right)\left(d \varepsilon^{2} / 2 \varepsilon+d \mu^{2} /\left(1-\mu^{2}\right)\right) \\
& +m^{2} \varepsilon^{-Q} \cdot 2 \varepsilon\left(1-\mu^{2}\right) d \varphi^{2} .
\end{aligned}
$$

Now since $Q=Q(\mu), \partial / \partial \varepsilon$ are not geodesics, although it is orthogonal to the singularity. If we define

$$
\xi=m\left(1-\mu^{2}\right)\left(Q^{2}-Q+1\right)^{-1} \varepsilon^{\left(Q^{2}-Q+1\right) / 2}
$$

which is the proper time of the $\partial / \partial \varepsilon$ curves for fixed $\mu$, we find that $d \xi=m\left(1-\mu^{2}\right) \frac{1}{2} \varepsilon^{\left(Q^{2}-Q-1\right) / 2} d \varepsilon+($ higher order terms). Thus although $(\xi, t, \mu, \varphi)$ does not define a Gaussian coordinate system based on the singularity at $\varepsilon=\xi=0$, its deviation from such a coordinate system goes to zero as $\varepsilon \rightarrow 0$, and the singularity structure obtained from using these two coordinate systems should be identical. Ignoring the cross terms in $d \xi d \mu$, we obtain the following singularity structure

$$
{ }_{0} d s^{2}=-{ }_{0} V^{2}(\mu) d t^{2}+{ }_{0} g_{\mu} d \mu^{2}+{ }_{0} g_{\varphi} d \varphi^{2}
$$

where ${ }_{0} V,{ }_{0} g_{\mu},{ }_{0} g_{\varphi}$ are some messy functions of $\mu$, and

$$
\boldsymbol{p}=\left(p_{0}, p_{+}, p_{-}\right) ; \quad \text { with } \gamma=\left(Q^{2}+1\right) /(Q-1)^{2} .
$$

From (43) we verify that as $Q=1, \gamma=\infty$, and $\lambda=1$ is the regular event horizon $(r=2 m)$ of the Schwarzschild solution. According to Erez and Rosen [12], if we let $Q=1+q_{l} P_{l}(\mu)$ (i.e., a monopole plus a single higher 
order pole term), the asymptotic behavior near infinity corresponds to a Newtonian $l^{\text {th }}$ pole, and $q_{l}$ can be identified as the corresponding $l^{\text {th }}$ order pole moment ${ }^{7}$. It is remarkable to see that $q_{l}$ is directly related to (in fact, uniquely determined by) $\gamma$, a parameter of the structure of the singularity itself!

The above construction, however, fails at $|\mu|=1$ since the coordinate $\mu$ itself is singular there. Using a different coordinate system, and taking appropriate limits with caution, we find that in this case

$$
\gamma(\mu= \pm 1)=\left(Q^{2}+2\right) /\left(Q^{2}-2 Q+2\right), \quad Q=Q(\mu= \pm 1) .
$$

Thus there is a discontinuous jump in the singularity extrinsic curvature, apparently related to the well known phenomenon of "directional singularity" [11]. There may be better ways of incorporating these end points.

(b) As an example of Weyl solutions with "ring-singularity" away from the $z$-axis of the Weyl coordinates, consider solutions of the Weyl equation in oblate spheroidal (O.S.) coordinates, which are related to the P.S. coordinates by the complex substitution

$$
\begin{gathered}
\lambda \rightarrow i \lambda, m \rightarrow i m \Rightarrow\left\{\begin{array}{l}
\varrho=m\left(\lambda^{2}+1\right)^{\frac{1}{2}}\left(1-\mu^{2}\right)^{\frac{1}{2}} \\
z=m \lambda \mu
\end{array}\right. \\
1 \geqq \mu \geqq 0 ; \quad \infty \geqq \lambda \geqq-\infty .
\end{gathered}
$$

The Weyl equation, which becomes

$$
\left(\left(\lambda^{2}+1\right) \psi_{, \lambda}\right)_{, \lambda}+\left(\left(1-\mu^{2}\right) \psi_{, \mu}\right)_{, \mu}=0
$$

has the general solution

$$
\psi=\sum_{l} a_{l} Q_{l}(i \lambda) P_{l}(\mu)
$$

$\chi$ is then generated from $\psi$ by

$$
\begin{aligned}
\chi_{, \lambda}= & \left(1-\mu^{2}\right)\left(\lambda^{2}+\mu^{2}\right)^{-1} \\
& \cdot\left\{\lambda\left(\lambda^{2}+1\right) \psi_{, \lambda}{ }^{2}-\lambda\left(1-\mu^{2}\right) \psi_{, \mu}{ }^{2}-2 \mu\left(\lambda^{2}+1\right) \psi_{, \lambda} \psi_{, \mu}\right\} \\
\chi_{, \mu}= & \left(1+\lambda^{2}\right)\left(\lambda^{2}+\mu^{2}\right)^{-1} \\
& \cdot\left\{\mu\left(\lambda^{2}+1\right) \psi_{, \lambda}{ }^{2}-\mu\left(1-\mu^{2}\right) \psi_{, \mu}{ }^{2}+2 \lambda\left(1-\mu^{2}\right) \psi_{, \lambda} \psi_{, \mu}\right\} .
\end{aligned}
$$

Metric (36) then reads

$$
\begin{aligned}
d s^{2}=-e^{2 \psi} d t^{2} & +m^{2} e^{2 \chi-2 \psi}\left(\lambda^{2}+\mu^{2}\right)\left(d \lambda^{2} /\left(\lambda^{2}+1\right)+d \mu^{2} /\left(1-\mu^{2}\right)\right) \\
& +m^{2} e^{-2 \psi}\left(\lambda^{2}+1\right)\left(1-\mu^{2}\right) d \varphi^{2} .
\end{aligned}
$$

7 This is obtained by expanding $\psi$ in Schwarzschild coordinates $(r / m-1=\lambda, \cos \theta=\mu)$ near $r=\infty$. We speculate, but have not checked, that it should also correspond to the $l^{\text {th }}$ moment as defined by Geroch [13]. 
Now a real solution $\psi$ made up of a finite sum in Eq. (46) is non-singular for all finite $\lambda$. It can then be checked that the only physical singularity of metric (47) is at $\lambda=\mu=0$, namely, a ring with radius $m$ centered on the $z$-axis

Define new coordinates

and

$$
\begin{aligned}
& R=\left(\lambda^{2}+\mu^{2}\right)^{-\frac{1}{2}} \Leftrightarrow \lambda=R /\left(1+u^{2}\right)^{\frac{1}{2}} \\
& u=\mu / \lambda \quad \Leftrightarrow \quad \mu=u R /\left(1+u^{2}\right)^{\frac{1}{2}} \\
& \infty \geqq R \geqq 0 ; \infty \geqq u \geqq-\infty ; R=0 \text { singularity . }
\end{aligned}
$$

Near $R=0$, Eq. (45b) give $\chi \simeq c \ln R$ where $c$ is in general some function of $u$. Taking the limit $\mu, \lambda \rightarrow 0$ and using Eq. (48), the first order metric near the singularity has the form

$$
d s^{2} \simeq-e^{2 \psi} d t^{2}+m^{2} e^{-2 \psi}\left[R^{2 c+2}\left(d R^{2}+R^{2}\left(1+u^{2}\right)^{-2} d u^{2}\right)+d \varphi^{2}\right]
$$

where $\psi$ is now some function of $u$ alone.

Thus the singularity metric is

$$
{ }_{0} d s^{2}=-{ }_{0} V^{2} d t^{2}+{ }_{0} g_{u} d u^{2}+{ }_{0} g_{\varphi} d \varphi^{2}
$$

where ${ }_{0} V(u)=e^{\psi(u)} ;{ }_{0} g_{\varphi}=m e^{-\psi(u)}$ and ${ }_{0} g_{u}$ is some messy function of $u$.

The extrinsic curvature is

$$
\boldsymbol{p}=(0,1,0) \Leftrightarrow \gamma=1 .
$$

(c) Another class of singular Weyl solutions are those singular at the origin of the Weyl coordinates. Consider the Weyl equation in spherical polar coordinates:

$$
r^{-2} \partial_{r}\left(r^{2} \partial_{r} \psi\right)+r^{-2} \sin ^{-2} \theta \partial_{\theta}\left(\sin ^{2} \theta \partial_{\theta} \psi\right)=0 .
$$

The general solution which goes to zero as $r \rightarrow \infty$ is of the form $\psi=\sum_{l} a_{l} P_{l}(\mu) r^{-(l+1)}$. $\psi$ 's made up of finite sums are singular at $r=0$.

However, in this case the metric components in general have singularities of the form $\exp \left(\alpha / r^{n}\right)$ which is not reducible to any power law behavior. Thus this type of Weyl solution, which we will call Curzon-like since the Curzon solution [14] is the simplest one of them, seems not to be normaldominated. The exact nature of their singularities merits further investigation.

\section{Singularity of the Kerr-Newman Metrics}

The above method of employing a singularity normal geodesic congruence to analyze the singularity structure turns out to have much wider applications beyond the class of normal-dominated static solutions. 
As an illustration, in this section we are going to use this method to obtain the invariant structure of the singularities of the Kerr-Newman stationary metrics [15]. It turns out that the structures obtained for the charged $(e \neq 0)$ and uncharged $(e=0)$ cases are qualitatively different. We therefore discuss them separately.

(a) The Kerr Metric $(e=0)$

$$
\begin{gathered}
d s^{2}=-(1-2 m r / \Sigma) d t^{2}+\Sigma\left(d r^{2} / \Delta+d \mu^{2} /\left(1-\mu^{2}\right)\right)+\left[\left(r^{2}+a^{2}\right)\left(1-\mu^{2}\right)\right. \\
\left.+2 m r a^{2}\left(1-\mu^{2}\right)^{2} / \Sigma\right] d \varphi^{2}+4 m r a\left(1-\mu^{2}\right) \Sigma^{-1} d \varphi d t \\
\Sigma \equiv r^{2}+a^{2} \mu^{2} ; \quad \Delta \equiv a^{2}+r^{2}-2 m r .
\end{gathered}
$$

Near the singularity $\Sigma=0(\Leftrightarrow r=0=\mu)$, the metric has the asymptotic form

$$
d s^{2} \simeq-d t^{2}+\Sigma a^{-2}\left(d r^{2}+a^{2} d \mu^{2}\right)+2 m r \Sigma^{-1} \omega^{2} ; \quad \omega \equiv d t+a d \varphi .
$$

Let us introduce new coordinates

$$
\begin{aligned}
& R=\frac{1}{2}\left(a \mu^{2}+r^{2} a^{-1}\right) \\
& \begin{array}{l}
\chi=r / a \mu \\
\Rightarrow d s^{2} \simeq-d t^{2}+d R^{2}+4 R^{2}\left(1+\chi^{2}\right)^{-2} d \chi^{2}+\sqrt{2} m \chi\left(a\left(1+\chi^{2}\right)\right)^{-\frac{1}{2}} \omega^{2} .
\end{array}
\end{aligned}
$$

Thus the singularity metric is

$$
{ }_{0} d s^{2}=-d t^{2}+4\left(1+\chi^{2}\right)^{-2} d \chi^{2}+\sqrt{2} m \chi\left(a R\left(1+\chi^{2}\right)\right)^{-\frac{1}{2}} \omega^{2}
$$

and the extrinsic curvature is $p=(0,1,-1 / 4)$. At $\chi=0$, (i.e., along $r=0$ ), the above procedure fails. However, going back to the original metric (51), we see that if we first let $r \rightarrow 0$ before taking the limit $\Sigma \rightarrow 0$, we have instead the asymptotic form

$$
d s^{2} \simeq-d t^{2}+\Sigma a^{-2}\left(d r^{2}+a^{2} d \mu^{2}\right)+a^{2} d \varphi^{2} .
$$

Thus the singularity structure at $\chi=0(r=0)$ is given by

$$
{ }_{0} d s^{2}=-d t^{2}+4 d \chi^{2}+a^{2} d \varphi^{2} ; \quad \boldsymbol{p}=(0,1,0) .
$$

The singularity structure suffers a discontinuous jump at the poles $(\chi=0)$. In any case, the topology of the singularity manifold is that of $T^{2}$ (torus) $\times R$.

(b) The Charged Kerr Metric $(e \neq 0)$

$$
\begin{aligned}
d s^{2}= & -\left(1-\left(2 m r-e^{2}\right) \Sigma^{-1}\right) d t^{2}+\Sigma\left(d r^{2} \Delta_{e}^{-1}+d \mu^{2} /\left(1-\mu^{2}\right)\right) \\
& +\left[\left(r^{2}+a^{2}\right)+a^{2}\left(2 m r-e^{2}\right) \Sigma^{-1}\left(1-\mu^{2}\right)\right] \\
& \cdot\left(1-\mu^{2}\right) d \varphi^{2}+2\left(2 m r-e^{2}\right) \Sigma^{-1} a\left(1-\mu^{2}\right) d \varphi d t,
\end{aligned}
$$


where

$$
\Delta_{e} \equiv e^{2}+a^{2}-2 m r+r^{2} \text {. }
$$

Near the singularity $\Sigma=0(\Leftrightarrow r=0=\mu)$, the metric has the asymptotic form

$$
d s^{2} \simeq-e^{2} \Sigma^{-1} \omega^{2}+\Sigma\left(d r^{2}\left(e^{2}+a^{2}\right)^{-1}+d \mu^{2}\right)+a^{2} d \varphi^{2}
$$

where

$$
\omega=d t+a d \varphi
$$

Introduce new coordinates

$$
R=\Sigma / 2 a ; \quad \chi=r \mu^{-\left(e^{2}+a^{2}\right) / a^{2}} .
$$

For $e \neq 0$, and $R \rightarrow 0$, we have

also

$$
r^{2} \simeq \chi^{2}(2 a R)^{\left(e^{2}+a^{2}\right) / a^{2}}+\text { higher order terms }
$$

$$
\mu^{2} \simeq 2 a R+\text { higher order terms } .
$$

With Eqs. (57), metric (56) becomes:

$$
\begin{aligned}
& d s^{2} \simeq-e^{2} \omega^{2}(2 a R)^{-1}+2 a R A^{-1}\left[d R^{2}+a^{2}\left(e^{2}+a^{2}\right)^{-1}(2 a R)^{1+\left(e^{2}+a^{2}\right) a^{-2}}\right] \\
& \text { where }+a^{2} d \varphi^{2} \\
& \qquad A=\left(2 a R+e^{2} r^{2} a^{-2}\right)
\end{aligned}
$$

Now since $e>0, r^{2} \ll R$ as $R \rightarrow 0$, so $A \simeq 2 a R$ as $R \rightarrow 0$ and the lowest order form of metric (58) is just

$d s^{2} \simeq-e^{2} \omega^{2}(2 a R)^{-1}+d R^{2}+a^{2}(2 a R)^{2+e^{2} a^{-2}}\left(e^{2}+a^{2}\right)^{-1} d \chi^{2}+a^{2} d \varphi^{2}$.

Thus the charged $\operatorname{Kerr}(e \neq 0)$ singularity has the structure

$$
\begin{gathered}
{ }_{0} d s^{2}=-e^{2} \omega^{2}(2 a)^{-1}+a^{2}(2 a)^{2+e^{2} a^{-2}}\left(e^{2}+a^{2}\right)^{-1} d \chi^{2}+a^{2} d \varphi^{2} . \\
p=\left(-1 / 2,1+e^{2}\left(2 a^{2}\right)^{-1}, 0\right) ; \quad T^{2} \times R
\end{gathered}
$$

which is qualitatively different from the uncharged case.

\section{Conclusions}

In this paper we have shown that a unique and invariant structure can be assigned to the class of static space-times which we call normaldominated. Two striking results are obtained concerning these singularities. First, we find that the dominant term of the Riemann tensor near the singularity is Type N. Whether this implies that the Weyl tensor may possess some special algebraic property near a generic singularity is not known. Second, we find that in general when $p_{+} \neq p_{-}$, 
the shear of null geodesic congruences near a normal-dominated static singularity seems to blow up at the same rate as the convergence. Thus, the general normal-dominated static singularity seems not to belong to the category investigated by Newman and Posadas.

In the analysis of exact static solutions, we find that the largest class of them are normal-dominated. In particular, the multipole fields of Erez and Rosen have their multipole structures directly related to the singularity structure. The normal-dominated singularity is a special case of a wider class which we would call "geodesic-normal singularity", which admits an irrotational geodesic congruence striking the singularity orthogonally. In this case one can also analyze the singularity along similar lines and assign to it an invariant structure. As an example, the singularity is worked out for the Kerr-Newman metrics.

The author is grateful to Professor E. T. Newman and Dr. D. Eardley for many helpful discussions.

\section{Appendix}

In this Appendix we compute the curvature components for the first order metric (16). It is straightforward to show that the curvature components for a general empty static space-time are [16]

$$
{ }^{(4)} R_{a b c d}=\varepsilon_{a b e} \varepsilon_{c d f}{ }^{(3)} R^{e f} ; \quad{ }^{(4)} R_{0 a b c}=0 ; \quad{ }^{(4)} R_{0 a 0 b}=V V_{\mid a b} .
$$

Using Eqs. (4)-(7) we find

$$
\begin{aligned}
{ }^{(4)} R_{a b c d}= & \varepsilon_{a b r} \varepsilon_{c d r}{ }^{(3)} R^{r r}+2 \varepsilon_{a b r} \varepsilon_{c d}{ }^{A(3)} R_{r A}+\varepsilon_{a b}{ }^{A} \varepsilon_{c d}{ }^{B}{ }^{(3)} R_{A B} \\
= & \varepsilon_{a b r} \varepsilon_{c d r}\left(K_{, r}+K^{A}{ }_{B} K^{B}{ }_{A}\right)+2 \varepsilon_{a b r} \varepsilon_{c d}{ }^{A}\left(K_{, A}-K^{B}{ }_{A \| B}\right) \\
& +\varepsilon_{a b A} \varepsilon_{c d}{ }^{B}\left({ }^{(2)} R^{A}{ }_{B}+K^{A}{ }_{B, r}+K K^{A}{ }_{B}\right) .
\end{aligned}
$$

Noting that $\varepsilon_{a b c}=\eta_{a b c} V-{ }^{(3)} g=\alpha \eta_{a b c}$, where $\eta_{123}=1$, this reduces to

$$
\begin{aligned}
& { }^{(4)} R_{a b c d}=\eta_{a b r} \eta_{c d r} \alpha^{2}\left(K_{, r}+K_{B}^{A} K_{A}^{B}\right)+\eta_{a b A} \eta_{c d B} g^{B C} \\
& \cdot \alpha^{2}\left({ }^{(2)} R_{C}^{A}+K_{C, r}^{A}+K K_{C}^{A}\right)+2 \eta_{a b r} \eta_{c d B} g^{B C} \alpha^{2}\left(K_{, C}-K_{C \| D}^{D}\right) .
\end{aligned}
$$

Also we have

$$
\begin{aligned}
{ }^{(4)} R_{0 r 0 r} & =V V_{, r r}, \\
{ }^{(4)} R_{0 r 0 A} & =V\left(V_{, r A}-V_{, C} K_{A}^{C}\right), \\
{ }^{(4)} R_{0 A 0 B} & =V\left(V_{\| A B}+V_{, r} K_{A B}\right),
\end{aligned}
$$

Putting solutions (10-16) into above we find for the lowest order terms of the curvature components: 


$$
\begin{aligned}
& R_{r-r-} \sim(1-\gamma) r^{-2+2 p_{-}} \quad \text { if } \quad \gamma \neq 1, R_{r-r-} \underset{\gamma=5}{\max ^{8}} r^{-8 / 3} \\
& \sim r^{-2} \quad \text { if } \gamma=1 \text {. } \\
& R_{r+r+} \sim(1-\gamma) r^{-2+2 p_{+}} \quad \text { if } \quad \gamma \neq 1, R_{r+r+} \underset{\gamma \rightarrow \infty}{\max } r^{-2<9} \\
& \sim \text { const if } \gamma=1 \text {. }
\end{aligned}
$$

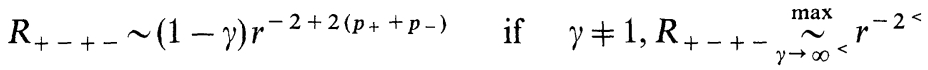

$$
\begin{aligned}
& =0 \quad \text { if } \quad \gamma=1 \text {. } \\
& R_{r+r-} \sim(\ln r)^{2} \\
& \sim \text { const } \\
& R_{r++-} \sim(\gamma,-)(\ln r) r^{-1+2 p_{+}} \quad \text { if } \quad \gamma,-\neq 0, R_{r++} \underset{\gamma \rightarrow \infty}{\max }(\ln r) r^{-1^{<}} \\
& \text {and } \gamma \neq 1 / 2 \\
& \sim r^{-1+2 p_{+}} \\
& \text {if } \quad \gamma,-=0, R_{r++} \underset{\gamma \rightarrow \infty}{\max } r^{-1<} \\
& \text { and } \gamma \neq 1 / 2 \\
& =0 \quad \text { if } \quad \gamma=1 / 2 \text {. } \\
& R_{r-+-} \sim\left(\gamma_{,+}\right)(\ln r) r^{-1+2 p_{-}} \quad \text { if } \quad \gamma_{,+} \neq 0, R_{r-+} \underset{\gamma=5}{\max }(\ln r) r^{-5 / 3} \\
& \text { and } \gamma \neq 1 / 2 \\
& \sim r^{-1+2 p-} \\
& \text { if } \gamma_{,+}=0, R_{r-+} \underset{\gamma=5}{\stackrel{\max }{\sim}} r^{-5 / 3} \\
& \text { and } \gamma \neq 1 / 2 \\
& =0 \text { if } \gamma=1 / 2 \text {. } \\
& R_{0 r 0 r} \sim(1-\gamma) r^{-4 /(1+\gamma)} \quad \text { if } \quad \gamma \neq 1, R_{0 r 0 r \underset{\gamma=\frac{1}{2}}{\max } r^{-8 / 3}} \\
& =0 \text { if } \gamma=1 \text {. } \\
& R_{0 r 0 \pm} \sim(1-\gamma)(\ln r) r^{-(3-\gamma) /(1+\gamma)} \text { if } \quad \gamma_{, \pm} \neq 0, \gamma \neq 1, R_{0 r 0 \pm} \underset{\gamma=\frac{1}{2}}{\max }(\ln r) r^{-5 / 3} \\
& \sim(1-\gamma) r^{-(3-\gamma) /(1+\gamma)} \quad \text { if } \quad \gamma_{, \pm}=0, \gamma \neq 1, R_{0 r 0 \pm} \underset{\gamma=\frac{1}{2}}{\max ^{-5 / 3}} \\
& \text { and }{ }_{0} V_{, \pm} \neq 0 \text {, } \\
& R_{0 r 0+} \sim r_{0}^{-1} V_{,+} ; R_{0 r 0-}=0 \quad \text { if } \quad \gamma=1 \text {. } \\
& R_{0+0+} \sim(1-\gamma) r^{-2 p-} \quad \text { if } \quad \gamma \neq 1, R_{0+0+} \underset{\gamma=\frac{1}{2}}{\sim} r^{-4 / 3} \\
& \sim \text { const if } \gamma=1 \text {, but }{ }_{0} V_{,+} \neq 0 \\
& =0 \quad \text { if } \gamma=1 \text { and }{ }_{0} V_{,+}=0
\end{aligned}
$$

${ }^{8}$ Here $A \stackrel{\text { max }}{\sim} r^{-n}$ means that the dominant term of $A$ as $r \rightarrow 0$ is of order $r^{-n}$ when we choose $\gamma$ so that $n$ is largest (i.e., the maximum blow-up rate of $A$ as $r \rightarrow 0$ ).

$9 n^{<}$means "arbitrary close to but strictly less than $n$ ". 


$$
\begin{aligned}
& R_{0-0-} \sim(1-\gamma) r^{-2 p_{+}} \quad \text { if } \quad \gamma \neq 1, R_{0-0-} \underset{\gamma=1<}{\max } r^{-2^{<}} \\
& \sim r^{-1} \quad \text { if } \gamma=1 \text { but }{ }_{0} V_{,+} \neq 0 \\
& \sim \text { const if } \gamma=1,{ }_{0} V_{,+}=0 \text { but }{ }_{0} V_{,-} \neq 0 \\
& =0 \quad \text { if } \gamma=1,{ }_{0} V_{, \pm}=0 \text {. } \\
& R_{0-0+} \sim(\gamma,-\gamma,+)\left(\ln ^{2} r\right) r^{2} p_{0} \quad \text { if } \quad \gamma_{, \pm} \neq 0, R_{0-0+} \underset{\gamma=\frac{1}{2}}{\max }(\ln r)^{2} r^{-2 / 3} \\
& \sim r^{2 p_{0}} \\
& \text { if } \gamma_{, \pm}=0, R_{0-0+} \underset{\gamma=\frac{1}{2}}{\stackrel{\max }{\sim}} r^{-2 / 3} \\
& \text { but }{ }_{0} V_{, \pm} \neq 0 \\
& =0 \\
& \text { if } \gamma_{, \pm}=0 \text { and }{ }_{0} V_{, \pm}=0 \text {. }
\end{aligned}
$$

We must emphasize that the above leading terms are computed from the first order metric (16). Even in the cases when the coefficients of the leading terms vanish, higher order terms will contribute in general.

\section{References}

1. Hawking, S., Penrose, R.: Proc. Roy. Soc. Lond. A 314, 529 (1970).

2. Hawking, S.: Phys. Rev. Lett. 15, 689 (1965); 17, 444 (1966); Proc. Roy. Soc. A 294, 511 (1966a); 295, 490 (1966b); 300, 187 (1967).

3. Penrose, R.: Phys. Rev. Lett. 14, 57 (1965).

4. Geroch, R.: Phys. Rev. Lett. 17, 445 (1966).

5. Belinskii, V.A., Khalatnikov,I.M.: Soviet Phys.-JETP 30, 1174 (1970). See also earlier papers cited in the references of their paper.

6. Eardley, D., Liang, E., Sachs, R.: J. Math. Phys. 23, 99 (1972).

7. Newman, E. T., Posadas, R.: Phys. Rev. 187, 1784 (1969).

8. Newman, E.T., Penrose, R.: J. Math. Phys. 3, 566 (1962).

9. Petrov, A.Z.: Einstein Spaces, p. 370, Oxford: Pergamon Press, 1969.

10. Taub, A. H.: Phys. Rev. 103, 454 (1956). We use slightly different coordinates here.

11. Godfrey, B.: "Static, Axisymmetric Vacuum Metrics," Ph. D. Thesis, Princeton University (1970, unpublished). See also Voorhees, B., "Axially Symmetric Distributions of Matter in General Relativity," Ph. D. Thesis, University of Texas (1971, unpublished).

12. Erez, G., Rosen, N.: Bull. Res. Coun. of Israel F 8, 47 (1959).

13. Geroch, R.: J. Math. Phys. 11, 2580 (1970).

14. Curzon, H.: Proc. Lond. Math. Soc. 23, 447 (1924).

15. We use the Boyer-Lindquist form here. Boyer, R., Lindquist, R.: J. Math. Phys. 8, 265 (1967); and Newman,E.T., Couch,E., Chinnapared, K., Exton, A., Prakash, A., Torrence, R.: J. Math. Phys. 6, 918 (1965).

16. See, for example, Israel, W.: Phys. Rev. 164, 1776 (1967).

E. P. T. Liang

Center for Relativity Theory

University of Texas

Austin, Texas 78712, USA 